\title{
ENERGETIC PARTICLE ANISOTROPIES AT THE HELIOSPHERIC BOUNDARY. II. TRANSIENT FEATURES AND RIGIDITY DEPENDENCE
}

\author{
V. Florinski ${ }^{1,2}$, E. C. Stone $^{3}$, A. C. Cummings ${ }^{3}$, and J. A. Le Roux ${ }^{1,2}$ \\ ${ }^{1}$ Department of Space Sciences, University of Alabama in Huntsville, Huntsville, AL 35899, USA \\ ${ }^{2}$ Center for Space Plasma and Aeronomic Research, University of Alabama in Huntsville, Huntsville, AL 35899, USA \\ ${ }^{3}$ Space Radiation Laboratory, California Institute of Technology, Pasadena, CA 91125, USA \\ Received 2014 October 29; accepted 2015 February 12; published 2015 April 13
}

\begin{abstract}
In the preceding paper, we showed that large second-order anisotropies of heliospheric ions measured by the Voyager 1 space probe during the August 2012 boundary crossing event could be explained by a magnetic shear across the heliopause preventing particles streaming along the magnetic field from escaping the inner heliosheath. According to Stone et al., the penetration distance of heliospheric ions into the outer heliosheath had a strong dependence on the particle's Larmor radius. By comparing hydrogen, helium, and oxygen ions with the same energy per nucleon, these authors argued that this effect must be attributed to larger cyclotron radii of heavier species rather than differences in velocity. We propose that gradient drift in a nonuniform magnetic field was the cause of both the large second-order anisotropies and the spatial differentiation based on the ion's rigidity. A latitudinal gradient of magnetic field strength of about 10\% per AU between 2012.7 and 2012.9 could have provided drift motion sufficient to match both LECP and CRS Voyager 1 observations. We explain the transient intensity dropout observed prior to the heliocliff using flux tube structures embedded in the heliosheath and magnetically connected to interstellar space. Finally, this paper reports a new indirect measurement of the plasma radial velocity at the heliopause on the basis of the time difference between two cosmic-ray telescopes measuring the same intensity dropout.
\end{abstract}

Key words: cosmic rays - ISM: magnetic fields - Sun: heliosphere - turbulence

\section{INTRODUCTION}

It is now generally accepted that the Voyager 1 space probe exited the heliosphere in 2012 August and is currently traveling through the region of space filled with plasma of an interstellar origin, known as the outer heliosheath, or OHS (Gurnett et al. 2013; Burlaga \& Ness 2014). The structure of the heliospheric boundary, deduced from magnetic field and energetic particle observations, proved to be quite complex. For example, galactic cosmic ray (GCR) fluxes have experienced two large increases between 2012.3 and the socalled heliocliff (HC) at 2012.65, which marked the large drop of particles of heliospheric origin, including anomalous cosmic rays (ACRs; Krimigis et al. 2013; Stone et al. 2013; Webber \& McDonald 2013). The magnetic field vector rotated through $180^{\circ}$ at 2012.57 in the last polarity reversal event observed by Voyager 1 (the structure named "CSO" in Burlaga \& Ness 2014), then experienced several short enhancements and drops before reaching the value of $0.45 \mathrm{nT}$ beyond the HC (Burlaga et al. 2013). Lower energy ion intensities showed characteristic dropouts that were clearly correlated with the magnetic field strength enhancements. The dropout regions were interpreted as flux tubes magnetically connected to the interstellar medium, perhaps as a result of an interchange plasma instability (Krimigis et al. 2013; Burlaga \& Ness 2014). Past the cliff, the low-energy ions, including ACRs, have declined dramatically to eventually fall below the instrument threshold. Ions streaming along the magnetic field were the first to disappear, while gyrating ions with pitch angles near $90^{\circ}$ were observed for several weeks after the boundary was crossed.

In the preceding paper (Florinski et al. 2013), we simulated the anisotropies of $5 \mathrm{MeV}$ protons near the heliopause using a simple transport model that included the effects of gyration, scattering, and field line random walk (FLRW) in a weakly turbulent magnetic field. The $\mathrm{HC}$ was represented by a magnetic shear layer with no change in magnetic field direction, but with a dramatic difference in the amplitudes of turbulent fluctuations on the two sides. We assumed weakly turbulent conditions in the inner heliosheath (IHS), and nearlaminar, scatter-free conditions in the OHS, based on Voyager results (Burlaga et al. 2013, 2014). The main feature of our model was the suppression of FLRW across the HC that led to a separation between the particles with pitch angles close to $90^{\circ}$, that were able to gyrate across the interface, and those traveling at smaller angles to the field, that remained trapped inside the IHS. That model could successfully explain the $\sim 5 \mathrm{MeV}$ ion spatial profile and anisotropies reported by Krimigis et al. (2013) using the LECP instrument on board Voyager 1.

Here we address a different set of observational results obtained using the CRS instrument (Stone et al. 2013). These authors compared temporal profiles of several ion species with similar energies per nucleon $\left(\sim 5 \mathrm{MeV} / \mathrm{n} \mathrm{H}^{+}\right.$and $\mathrm{He}^{+}$and $\sim 9 \mathrm{MeV} / \mathrm{n} \mathrm{O}^{+}$), therefore, having approximately the same velocity, but very different gyro-radii because of the mass difference. Their analysis clearly showed a dependence of the length of the post-HC decrease on rigidity. Such an observation would be difficult to explain in a model that relies on FLRW as the primary cross-field transport mechanism in the OHS. In fact, in the (admittedly, very simple) model of Florinski et al. (2013), the perpendicular diffusion length was independent of rigidity. While more realistic theoretical perpendicular diffusion models do exhibit a dependence on rigidity (e.g., Shalchi 2006), there is a much more efficient mechanism capable of discriminating between streaming and gyrating particles-the gradient drift in a nonuniform magnetic field. This is the basis of the model proposed here. 
One could readily estimate the required degree of magnetic field nonuniformity using the standard expression for gradient drift velocity,

$$
\boldsymbol{v}_{d}=\frac{m v_{\perp}^{2} c}{2 q B} \frac{\boldsymbol{B} \times \nabla B}{B^{2}} \sim \frac{r_{g}}{2 L} v_{\perp},
$$

where $\boldsymbol{B}$ is magnetic field, $q$ is the ion's charge, $\left.v_{\perp}=v_{\left(1-\mu^{2}\right.}\right)^{1 / 2}$ is its perpendicular velocity component, $\mu$ is the pitch-angle cosine, $r_{g}$ is the Larmor radius, and $L$ is the characteristic length associated with the gradient of $\boldsymbol{B}$ in the latitudinal direction. For the magnetic field primarily in the $-T$ direction, as observed, the field strength must increase in the southern direction so that $\boldsymbol{v}_{d}$ points radially outward for positively charged ions. Voyager 1 is north of the "nose" of the heliopause, where the magnetic field experiences the most compression; therefore, a southern gradient is expected. The factor of $1-\mu^{2}$ in Equation (1) strongly favors gyrating particles with $\mu \approx 0$.

The radial drift velocity component must be sufficient for the particles to move a distance $\Delta r$ in the radial direction, the measured OHS penetration length (about $1 \mathrm{AU}$ for $9 \mathrm{MeV} / \mathrm{n}$ singly charged oxygen; see Section 2) before escaping into the local interstellar medium (LISM) along the magnetic field line, i.e.,

$$
\frac{z_{\max }}{v_{\|}} \sim \frac{\Delta r}{v_{d}}
$$

where $v_{\|}=v \mu$ and $z_{\max } \sim 50-100 \mathrm{AU}$ is the escape distance of Florinski et al. (2013), $z$ being the direction of the magnetic field. Then, from Equations (1) and (2), we obtain $L \sim r_{g} z_{\max } /(2 \Delta r)=3 \mathrm{AU}$. The number is actually the lower limit on $L$ because we ignored the pitch angle factor $v_{\perp} / v_{\|}$, which is large for ions gyrating near $90^{\circ}$. The simple estimate above suggests that a magnetic field strength gradient of about $30 \%$ per $\mathrm{AU}$ is required to reproduce the measured width of the OHS penetration region. In fact, as we show below, using numerical simulations, a much more moderate gradient of under $10 \%$ per AU is sufficient to obtain a width of the ACR ramp consistent with the observations.

\section{VOYAGER OBSERVATIONS}

The observations reported here were performed with the LET detector, which is part of the CRS instrument on board Voyager 1 (Stone et al. 1977). Figure 1 shows the count rate of galactic electrons and intensities of three heliospheric ion species measured by Voyager 1 during the second half of 2012. With the exception of the electron count rates, the figure contains essentially the same data as Figure 2 of Stone et al. (2013). Galactic electrons are seen to respond rapidly to the magnetic field changes associated with the heliosheath particle dropouts. This is exactly the behavior expected inside magnetic flux tubes connected to the LISM. Because the electrons are relativistic and scattering is weak or absent, they quickly fill the interior of the tube where their intensity is very close to interstellar (beyond the HC, shown with a dotted line). The ions shown in the figure travel slower $(0.1 c)$, and their Larmor radius is larger; therefore their transport is not entirely along the magnetic field. As argued in Florinski et al. (2013), gyration effects allow ions to overcome magnetic shear at the $\mathrm{HC}$ and leak into the LISM - the same process is operating at the boundaries of the two flux tubes.

The species in Figure 1 are arranged in order of increasing rigidity, from top to bottom. There is a visible trend of increasing width of the post-HC ramp with rigidity. Whereas hydrogen ions persisted for about two weeks, higher rigidity anomalous oxygen ions were detectable for almost three months until 2012.9, the time of arrival of a shock or pressure wave (Burlaga et al. 2013). This corresponds to about $0.9 \mathrm{AU}$ traveled at the speed of Voyager 1. After the ACRs have disappeared, GCRs remained the only energetic particle components measured by the CRS. Their flux is about $1 \%$ of the ACR intensity in the IHS in the energy channels shown in Figure 1.

Ions are also differentiated based on their pitch angle. For the period of time near the HC, the LET C and D centers of view were directed at $47^{\circ}$ and $105^{\circ}$ with respect to the magnetic field, respectively. Both telescopes have an opening angle of $50^{\circ}$. As a result, the former measured both streaming and gyrating particles, while the latter primarily measured those gyrating near $90^{\circ}$ pitch angle. In each panel, gyrating particles decline at a slower rate than streaming. As we show here, gradient drift can account for intensity dependence on both rigidity and pitch angle.

We now examine more closely the second (longer lasting) dropout in heliospheric particle intensities (days 226 to 233). Figure 2 plots count rates of $2-8 \mathrm{MeV}$ protons from LET telescopes B and D. The most striking feature of this figure is the displacement in time of the intensity profiles between the two telescopes, with LET B trailing LET D by 12-14 hours. We explain this feature by the difference in the gyrophase (rather than pitch angle) of the ions measured by the two telescopes. As explained in detail in Section 4, the orientation of LET B is such that gyrating ions reach it from a point of space that is closer to the Sun by about one Larmor radius, while LET D measures ions arriving from a point farther from the Sun by approximately the same distance. The time displacement is then a result of a radial gradient in the density of guiding centers inside the dropouts, a manifestation of the well-known circulating current anisotropy (Parker 1957; Axford 1965). Because this time difference is approximately equal to the spacecraft gyro-orbit crossing time, we use this measurement in Section 5 to deduce the radial component of plasma speed inside the second dropout. As we show there, the latter had to be very small because the time delay is too close to what would be expected based on spacecraft motion alone.

\section{THE PROBLEM GEOMETRY}

We will not be concerned here with the global structure of the heliosphere during the Voyager 1 encounter of the heliopause. Such large-scale simulations could potentially explain the unexpectedly early crossing of the heliopause through a wavy, turbulent nature of the interface (Borovikov \& Pogorelov 2014). Another outstanding issue is the close alignment between the magnetic fields on the two sides of the heliopause. Again, it is possible that some kind of instability helps align the fields (Swisdak et al. 2013; Strumik et al. 2014). Following Krimigis et al. (2013), we interpret the particle dropouts as the regions interior to LISM-connected magnetic flux tubes that have sunk into the heliosheath, possibly as a result of a pressure-driven "ballooning" instability 


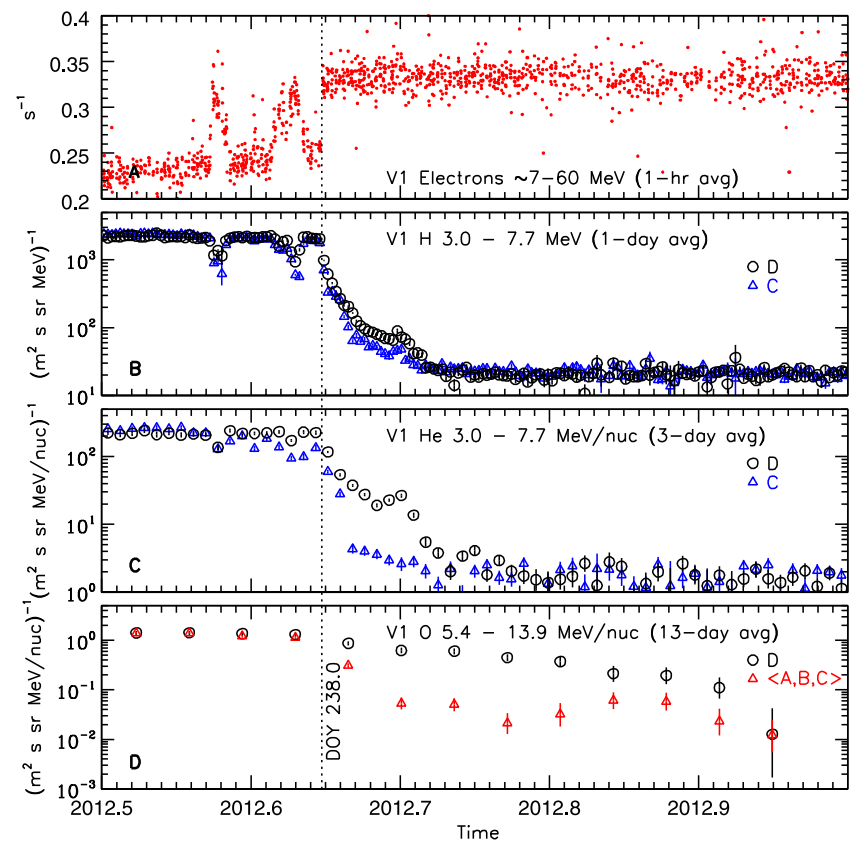

Figure 1. From top to bottom: intensities of electrons, hydrogen, helium, and oxygen ions at Voyager 1 during the second half of 2012. The difference in intensities between the LET detectors is a result of large second-order anisotropy of the ion distributions.

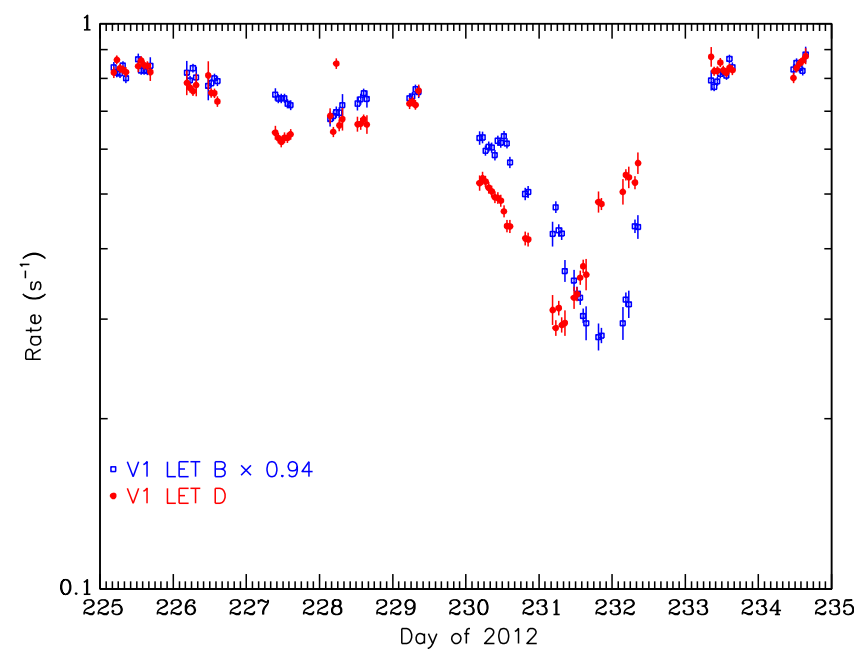

Figure 2. Count rates of $2-8 \mathrm{MeV}$ protons around the time of the second intensity dropout from two of the four LET telescopes. LET B (blue) measures ions with guiding centers behind Voyager 1 (toward the Sun), while LET D (red) measures ions gyrating in from a farther distance from the Sun.

(e.g., Sakai 1982; Viñas and Madden 1986). The magnetic pressure has increased by more than a factor of four across the heliopause, and it is likely that the gas kinetic pressure (which includes the energetic particle pressure) has dropped by a similar amount to maintain the overall equilibrium. One could estimate the growth rate of the ballooning instability by the magnetic Brunt-Väisälä frequency $\Omega_{\mathrm{BV}}$, which is the MHD analog of gravity wave frequency in a stratified media subjected to a volume force in the direction perpendicular to the density gradient. The magnetic field line tension plays the role of gravitational force. From Viñas and Madden (1986), we

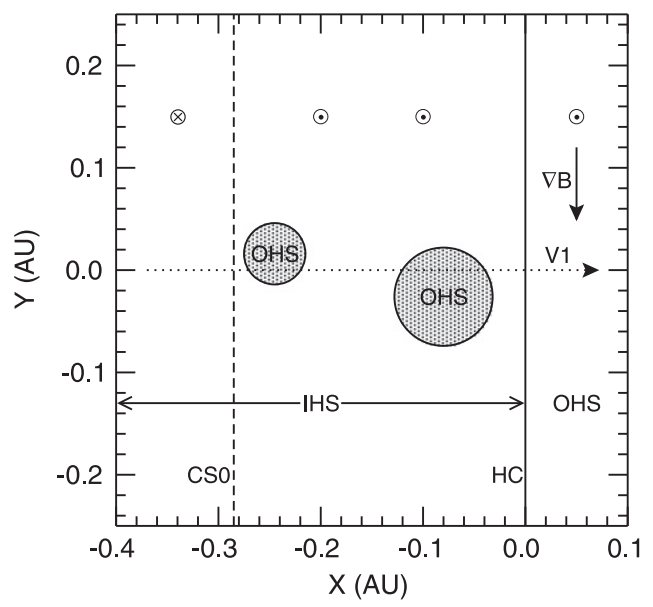

Figure 3. Model geometry showing the last observed current sheet CSO, two interstellar magnetic flux tubes (shaded circles) embedded in the inner heliosheath, and the heliocliff (HC) interface. The magnetic field is in the $\pm \hat{z}$ direction. The straight dotted line at $y=0$ is the Voyager 1 trajectory.

may write, approximately,

$$
\Omega_{\mathrm{BV}}^{2} \approx \frac{1}{r} \frac{v_{a}^{2} c_{s}^{2}}{v_{a}^{2}+c_{s}^{2}}\left(\frac{2}{\gamma} \frac{d \ln p}{d r}-\frac{d \ln B}{d r}\right),
$$

where $p$ is the plasma pressure (including the pickup-ion contribution), $v_{a}$ is the Alfvén speed, and $c_{s}$ is the sound speed. In writing out Equation (3), it was assumed that $L \ll r$, where $r=122 \mathrm{AU}$ is the distance to the heliopause.

The instability will develop if (a) the magnetic field lines are convex toward the interstellar side, and (b) the expression on the right hand side of (3) is negative, so that $\Omega_{\mathrm{BV}}$ is imaginary. Because the pressure drops and the magnetic field increases with radial distance at the heliopause, the second criterion is always satisfied. Taking the number density, effective temperature, and magnetic field of $0.002 \mathrm{~cm}^{-3}, 1.4 \times 10^{6} \mathrm{~K}$, and $0.2 \mathrm{nT}$ on the heliosheath side, and $0.05 \mathrm{~cm}^{-3}, 2 \times 10^{4} \mathrm{~K}$, and $0.4 \mathrm{nT}$ on the interstellar side, respectively, and the transition width of $0.1 \mathrm{AU}$, we obtain $\left|\Omega_{\mathrm{BV}}\right| \sim 2 \times 10^{-7} \mathrm{~s}$ using the heliosheath values of $v_{a}$ and $c_{s}$ that provide a lower instability threshold, so the characteristic time for the instability to grow is of the order of two months. This appears to have the right order of magnitude compared with the duration of the heliopause encounter. A faster growth rate could be obtained if the curvature of the field lines is locally larger than $r^{-1}$, perhaps due to a wave traveling along the surface of the heliopause (e.g., Florinski et al. 2005; Borovikov et al. 2008), or for a narrower transition.

Figure 3 illustrates the model geometry adopted for this work. The coordinate system chosen is such that the $x, y$, and $z$ directions correspond, approximately, to the $R, N$, and $-T$ directions of the spacecraft RTN frame. To the left of the last magnetic polarity reversal interface $\mathrm{CSO}$, the plasma has all the properties of IHS. Between CSO and the HC, we introduced two cylindrical flux tubes (shaded circles) that are filled with OHS plasma that has sunk into the IHS as a result of instability. Other flux tubes are likely to be present in this region, but we have no information about them. In this figure, the magnetic field strength increases observed by Voyager 1 correspond to boundaries of the flux tubes that are magnetically connected to the OHS and are therefore depleted in heliospheric energetic 
Table 1

Transport Model Parameters Compared with Florinski et al. (2013)

\begin{tabular}{|c|c|c|c|c|c|c|c|}
\hline & $B^{\mathrm{ih}}, \mathrm{nT}$ & $B^{\mathrm{oh}}, \mathrm{nT}$ & $D_{0}^{\mathrm{ih}}, \mathrm{s}^{-1}$ & $D_{0}^{\mathrm{oh}}, \mathrm{s}^{-1}$ & $K^{\mathrm{ih}}, \mathrm{AU}$ & $K^{\mathrm{oh}}, \mathrm{AU}$ & $z_{\max }, \mathrm{AU}$ \\
\hline Florinski et al. (2013) & 0.3 & 0.4 & $10^{-4}$ & $2 \times 10^{-7}$ & $6 \times 10^{-4}$ & $5 \times 10^{-5}$ & 70 \\
\hline This work & 0.25 & 0.45 & $10^{-4}$ & $10^{-6}$ & $6 \times 10^{-4}$ & $5 \times 10^{-6}$ & 60 \\
\hline Constrained? & V1 MF & V1 MF & V1 SD & $<\mathrm{V} 1 \mathrm{SD}$ & no & no & $<r$ \\
\hline
\end{tabular}

particles. We assume that the tubes possess all the properties of the LISM magnetic field, including the nearly zero short scale turbulent component (by short scale here we mean turbulent fluctuations of the size relevant to the transport of ions with energies below $\sim 100 \mathrm{MeV} / \mathrm{n}$ ). The magnetic field is into the page (in the $T$ direction) to the left of CSO and out of the page in the remainder of the IHS and in the OHS. We ignored the non-azimuthal field components for the sake of simplicity. To the right of the $\mathrm{HC}$, we imposed a gradient of magnetic field strength in the $-\hat{\boldsymbol{y}}$ (latitudinal) direction.

Using the nominal Voyager 1 speed of $17.03 \mathrm{~km} \mathrm{~s}^{-1}$ and zero average radial plasma flow, we can estimate the radial extent of the two depleted flux tubes at $0.05 \mathrm{AU}$ and $0.08 \mathrm{AU}$, respectively, based on magnetic field observations. The "connection length" $z_{\max }$ of Florinski et al. (2013) can also be interpreted as the flux tube length. Here we used $z_{\max }=60$ AU. Because it is unlikely that Voyager 1 crossed the tubes precisely along their diameters, we used wider flux tubes $(0.06$ and $0.096 \mathrm{AU}$, respectively) that were displaced in the latitudinal direction relative to the path of the spacecraft. The flux tubes are connected to the LISM at both ends.

The transport model used here is essentially the same as in Florinski et al. (2013). We integrate the trajectories of the Boltzmann equation in the time-backward sense from the detection point, until the boundary of the simulation box is reached. If $D(\boldsymbol{r}, \boldsymbol{v})$ is the isotropic pitch-angle scattering coefficient and $K(\boldsymbol{r})$ is the magnetic FLRW coefficient $K$ (Jokipii \& Parker 1969), then the transport equation for the particle's phase space density $f(\boldsymbol{r}, \boldsymbol{v})$ can be written as

$$
\begin{gathered}
\frac{\partial f}{\partial t}+\boldsymbol{v}_{\|} \cdot \nabla_{\|} f+\left(\boldsymbol{v}_{\perp}-\left|v_{\|}\right| \nabla_{\perp} K\right) \cdot \nabla_{\perp} f \\
-\left|v_{\|}\right| K \nabla_{\perp}^{2} f-\Omega \frac{\partial f}{\partial \varphi}-D \nabla_{\theta \varphi}^{2} f=0,
\end{gathered}
$$

where $\phi$ is gyrophase, $\nabla_{\|}$and $\nabla_{\perp}$ are the gradient operators in the directions parallel and perpendicular to $\boldsymbol{B}$, respectively, and $\nabla_{\theta \varphi}$ is the gradient in velocity space at $v=$ const. The coefficient $K$ is related to the perpendicular diffusion coefficient as shown in Florinski et al. (2013). The gradient $\nabla_{\perp} K$ is zero everywhere except in the flux tube walls and at the $\mathrm{HC}$, both of which are assumed to have a thickness of $0.005 \mathrm{AU}$.

Because the model will be applied to different ion species, we postulate the following expression for the scattering coefficient, motivated by quasi-linear theory (Jokipii \& Parker 1969). For isotropic scattering, the expression is particularly simple,

$$
D=\Omega \frac{\left\langle\delta B^{2}\right\rangle}{B^{2}}=D_{0} \frac{B}{B_{0}} \frac{Q}{\gamma A},
$$

where $Q$ is the charge number, $A$ is the mass number, $\gamma$ is the relativistic factor, and $D_{0}$ is the reference value of the scattering coefficient that contains the dependence on the turbulent power $\left\langle\delta B^{2}\right\rangle$, which has different values in the IHS and OHS (Florinski et al. 2013). For the magnetic field, we used a reference value $B_{0}=0.45 \mathrm{nT}$.

Table 1 compares the model parameters used in this paper with Florinski et al. (2013). The superscripts "ih" and "oh" refer to the inner and the outer heliosheaths, respectively. Most of these parameters were determined, or at least constrained, by in situ observations and other models, as indicated on the bottom line of Table 1. The magnetic field values were taken from Burlaga et al. (2013). The scattering rates were estimated using $\left\langle\delta B^{2}\right\rangle / B^{2}$, reported by Burlaga et al. (2014) in Equation (5). For the IHS, this was done based on the actual measurements of the standard deviation of the components of $\boldsymbol{B}$, while for the OHS we used their upper limit on the turbulent intensity $\left\langle\delta B^{2}\right\rangle / B^{2}<2.8 \times 10^{-4}$, which implies that $D^{\text {oh }}<10^{-5} \mathrm{~s}^{-1}$, and is probably much less. Both the old and the new values in column 5 of Table 1 satisfy this condition. Tests showed that the model is not sensitive to the value of the scattering rate beyond the heliopause, as long as it is sufficiently small (if this were not the case, the large anisotropy would not persist well into the OHS region). The larger value of $D^{\text {oh }}$ provided a better agreement with CRS He and $\mathrm{O}$ data (Stone et al. 2013) that were not included in our 2013 study. The flux tube length $z_{\max }$ must be less than the size of the heliosphere $r$, which is of the order of $100 \mathrm{AU}$.

The magnetic FLRW coefficients $K^{\text {ih }}$ and $K^{\text {oh }}$ determine the rate of cross-field diffusion and are essentially free parameters in the model. Following Florinski et al. (2013), we have chosen the values that are small enough to retain sharp features in the radial intensity profile (the dropouts and the $\mathrm{HC}$ itself) similar to the observed. Owing to a significantly more complicated geometry of the new model compared with the previous paper, FLRW suppression regions were not included here. Because our model requires that field line mixing does not occur across the magnetic boundaries, to avoid the difficult numerical task of adding FLRW suppression regions around the circular flux tubes, we simply reduced the value of $K^{\mathrm{oh}}$ by a factor of 10 (column 7 of Table 1). Numerical tests showed that, without this reduction, particle transport across the magnetic shear is too efficient, at least in the case of protons, washing out the sharp features of the intensity dropouts and the $\mathrm{HC}$ itself. The conclusions of the paper are not affected by this change because pitch-angle separation is now achieved primarily via gradient drift.

The strength of the magnetic field in the model was increasing linearly in the OHS in the $-\hat{\boldsymbol{y}}$ (southward) direction with a gradient of $0.038 \mathrm{nT}$ per $\mathrm{AU}$, which corresponds to $L \sim 12$ AU. This is four times larger than the estimate in Section 1 , so the required magnetic field strength gradient in the latitudinal direction is relatively modest. The gradient could be a local feature developed as a result of instability, rather than a part of the persistent large-scale structure of the heliopause. Voyager 1 measured a radial gradient (decrease) of $|\boldsymbol{B}|$ of $\sim 15 \%$ per AU between the $\mathrm{HC}$ and the pressure wave (Burlaga 


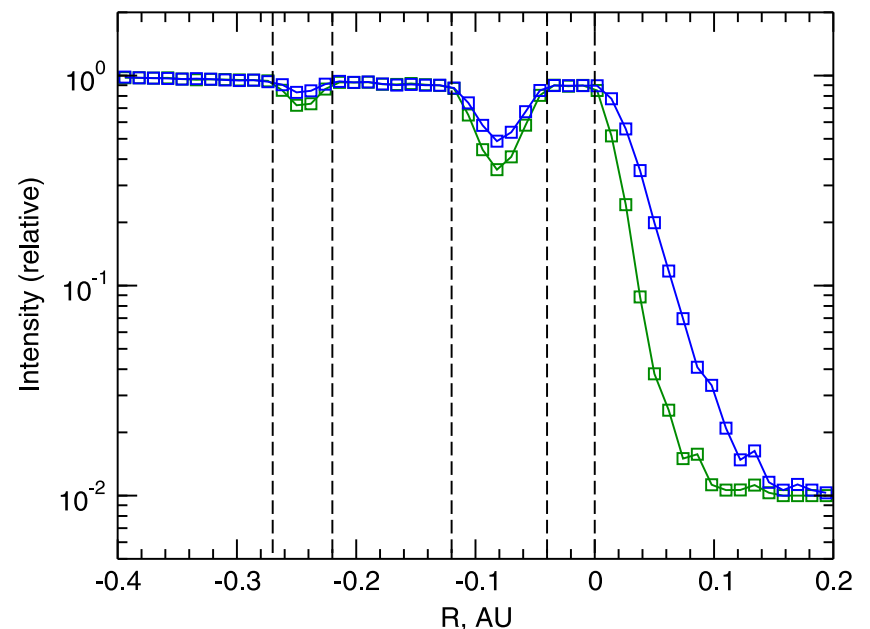

Figure 4. Simulated intensity of $5 \mathrm{MeV}$ protons in the vicinity of the heliocliff. The green symbols show the radial profile for $\mu=1$ (streaming) and the blue symbols show the same for $\mu=0$ (gyrating) ions. A 1\% GCR flux was added to both quantities. Vertical dashed lines mark the flux tube crossing points and the heliocliff boundary.

\& Ness 2014), and a comparable latitudinal gradient could have been present during that period.

\section{INTENSITY DROPOUTS AND RAMP WIDTHS}

Two prominent intensity dropouts were measured by Voyager 1 with the first occurring about a month before the HC. In our model, the dropouts correspond to the time intervals when Voyager 1 was inside the magnetic flux tubes connected to the OHS at both ends (see Figure 3). Because of fast, nearly scatter-free transport, heliospheric ions would quickly empty into the LISM with the ions gyrating near $\mu=0$ left behind, as observed.

Figure 4 shows the simulated dropout in $5 \mathrm{MeV} \mathrm{H}^{+}$ions. The boundaries of the flux tubes that correspond to the energetic particle dropouts are shown with vertical lines in this figure. The depths of the simulated dropouts is similar, but somewhat less than observed, which is most likely because we did not include the FLRW suppression regions at the flux tubes boundaries. Note that the intensities recover almost completely between the dropouts, which is again consistent with both LECP and CRS observations (Krimigis et al. 2013; Stone et al. 2013). Gyrating particles experience a weaker intensity change, as one would expect. Past the $\mathrm{HC}$, gyrating protons (blue lines) are more efficiently transported by the gradient drift and their ramp is therefore wider than for the streaming protons (green). To facilitate comparison with Voyager CRS observations (Figure 1), we have added a small GCR component (1\% of ACR intensity in the heliosheath) to the simulated intensity.

Next, we examine the extents of the post-HC intensity decreases for different ion species. Results for $\mathrm{He}^{+}$and $\mathrm{O}^{+}$ $5 \mathrm{MeV} / \mathrm{n}$ ACRs are shown in Figure 5 for pitch angles of 30, 60 , and 90 degrees. As with hydrogen, a constant 1\% GCR flux was added to the computed helium and oxygen intensities in Figure 5. The dropouts are barely visible for helium and absent for oxygen, as observed. Like protons, these ions have large second-order (pancake) anisotropies in the OHS. Gyrating oxygen ions persist for almost $1 \mathrm{AU}$ into the OHS, while the ramp in gyrating helium ion intensity extends out to about
0.4 AU (compare to the width of the $\mathrm{H}^{+}$ramp of $0.1 \mathrm{AU}$ from Figure 4). These numbers are mostly consistent with the observations (Figure 1).

The above result demonstrates that, in the near absence of pitch angle scattering, gradient drift can spatially separate particles based on their radius of gyration. In other words, ions are differentiated by a combination of their charge per nucleon, energy per nucleon, and pitch angle. For singly charged ions with the same energy per nucleon, only charge and pitch angle separation occurs with particles with lower charge/n (oxygen) and pitch angles close to $90^{\circ}$ penetrating the farthest into the OHS region, while those with high charge/n (protons) streaming along the magnetic field disappearing soon after the crossing. Unlike the solar magnetic field, the interstellar field is nearly devoid of turbulent fluctuations (Burlaga et al. 2014) so that coherent processes such as scatter-free streaming and gradient/curvature drift are expected to play a major role in the transport of low-energy ions in the OHS and LISM.

We now turn our attention to the observed circulating current anisotropy (see Section 2 for observational context), which is produced by the gyromotion of an ensemble of particles with a density gradient normal to the mean magnetic field. This anisotropy is perpendicular to both $\boldsymbol{B}$ and $\nabla f$, and is therefore in the $y$ direction. As a simple example, consider two particle detectors, one pointing in the $N$ direction, and the other in the $-N$ direction. Figure 6 demonstrates the physics of the anisotropy produced in this pair of detectors (also known as diamagnetic drift effect) as a space probe enters a magnetic flux tube with a negative intensity gradient (depletion region) from left to right. For the magnetic field, as shown in Figure 3, ions with an upward directed velocity vector represent conditions $\sim r_{g}$ ahead of the spacecraft, so they would be first to experience a dropout and the subsequent recovery. The opposite is true for ions traveling in the downward direction. Because the density of guiding centers is higher in the heliosheath, the excess creates a current in the downward direction, even though there is no net drifting of particles in that direction. This current would produce a first-order anisotropy in a pair of detectors pointing up and down in this figure.

Because our model retains particle gyromotion, it can readily reproduce the gyration-induced anisotropy. Simulations were performed in the region around the second flux tube for protons whose velocity vectors were either parallel or antiparallel to the $y$ axis at detection. Figure 7 compares the simulated intensity profiles of hydrogen ions with a pitch angle of $90^{\circ}$ and gyrophase angles of $90^{\circ}$ and $270^{\circ}$ corresponding to the up and down directions in Figure 6, respectively. The boundaries of the second magnetic flux tube are shown with vertical dashed lines. Intensity reaches $50 \%$ of its value in the heliosheath at the bottom of the dropouts.

Figure 7 shows that the downward intensity lags behind the upward intensity, as one would expect from the preceding analysis (see Figure 2). The spatial displacement is $2 r_{g}$, or $0.01 \mathrm{AU}$ for hydrogen. Examination of the figure shows that the particle intensity dropout does not start exactly at the magnetic boundary, but is delayed by about one $r_{g}$; likewise, the recovery is complete when the spacecraft is still inside the tube, one $r_{g}$ from the boundary. This is because ions with gyrocenters less than 1 gyroradius from the edge of the tube will re-enter the IHS each gyration rather than escaping along 


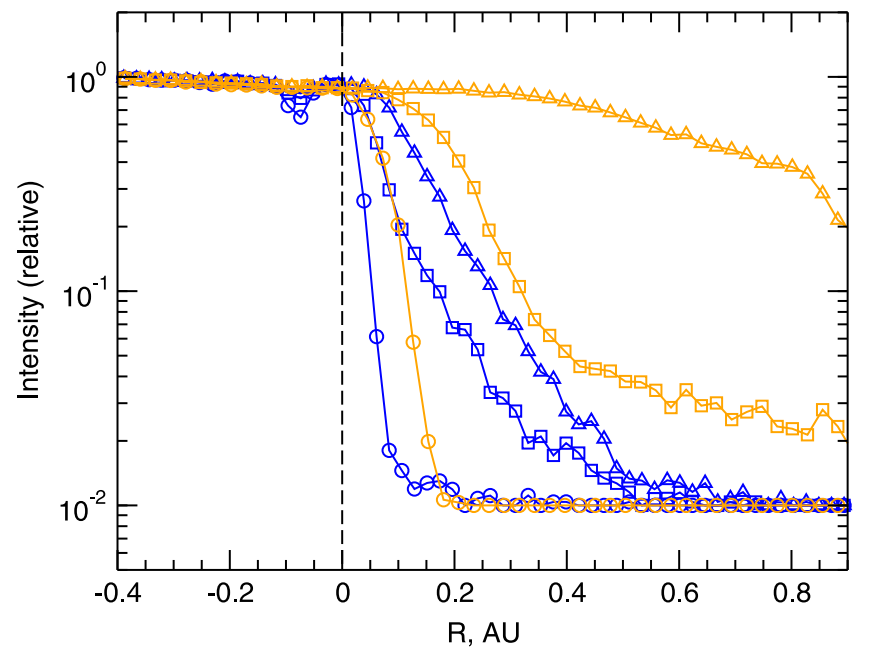

Figure 5. Same as in Figure 4, but for $5 \mathrm{MeV} / \mathrm{n} \mathrm{He}^{+}$(blue) and $5 \mathrm{MeV} / \mathrm{n} \mathrm{O}^{+}$ (orange). Circles are intensities of particle with the final pitch angle of $30^{\circ}$, squares $60^{\circ}$, and triangles $90^{\circ}$. The heliocliff is shown with a vertical dashed line.

the magnetic field into the LISM; consequently their intensity is essentially the same as that in the heliosheath.

For a moving spacecraft, the temporal shift would be affected by the motion of the plasma background that carries the magnetic field. The shift, therefore, provides an independent estimate of the plasma velocity when a direct measurement is not possible, such as on Voyager 1 with its nonoperational plasma detector. We perform this analysis taking into account the geometry of the LET telescopes and the measured direction of the magnetic field in the following section.

\section{TIME DELAY ANALYSIS}

Here we theoretically compute the time difference in the detection of the energetic particle intensity changes in the second ACR intensity dropout between LET telescopes B and D (see Figure 2). Figure 8 illustrates the geometry used in this analysis. The magnetic field vector is normal to the page, which is therefore the plane of gyration; all vectors are shown in projection onto that plane. During the time of interest, the magnetic field was $\boldsymbol{B}=(0.112,-0.391,0.124) \mathrm{nT}$, in the spacecraft's RTN coordinate frame (see Table 1 of Burlaga et al. 2013). In the figure, $\alpha$ is the angle, measured clockwise, between the projected LET view direction and the direction of travel of the spacecraft, which is very close to radial. The intensity gradient $\nabla f$ is assumed to lie in the gyration plane, as expected for the flux tube scenario, where the particle gradient is normal to the surface of the tube. However, the direction of the gradient in the plane is not known; we assume here that it forms an angle $\beta$ with the radial direction. Dashed lines are planes of constant intensity. The projected Larmor radius is $r_{g} \sin \gamma$, where $\gamma$ is the angle between $\boldsymbol{B}$ and the view direction. The circular arc in Figure 8 is a trajectory of a positively charged ion impacting on the instrument sensor.

The spacecraft detects ions whose guiding centers are at the position marked "GC" in the figure. The instrument, as shown, therefore measures the particle intensity at a point labelled " $\mathrm{V}_{1}{ }_{1}$ " (behind, along the trajectory). A straightforward calculation shows that the time passed on the spacecraft between the point of intensity change due to a gradient (the

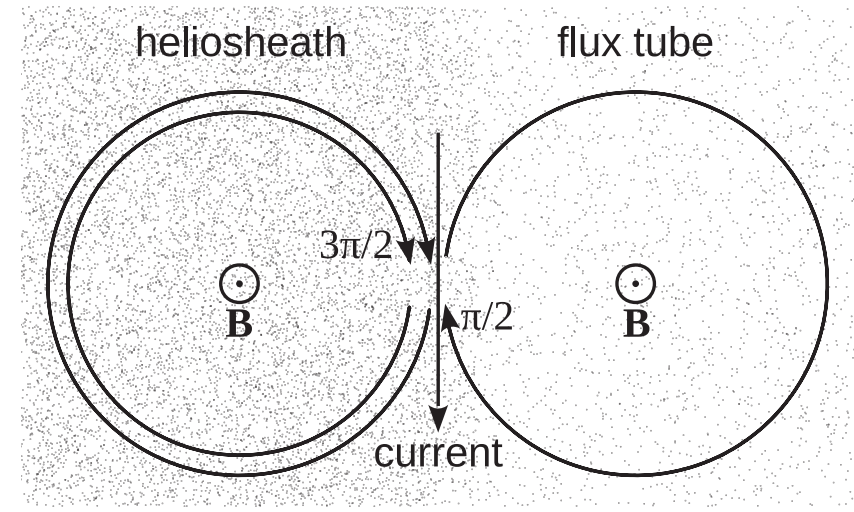

Figure 6. Orbits of positively charged particles near a wall of a flux tube. The number of energetic particles per unit volume is proportional to the density of dots. A current is produced in the downward direction $(\sim \boldsymbol{B} \times \nabla f)$ because there are more orbits to the left of the observer than to the right.

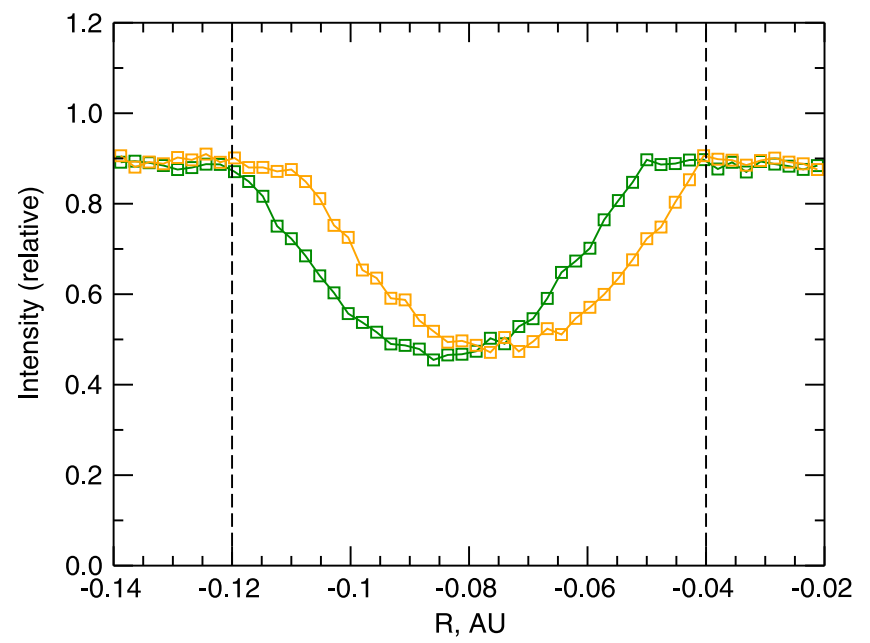

Figure 7. Simulated radial intensity of $5 \mathrm{MeV}$ protons with a pitch angle of $90^{\circ}$ and a gyrophase angle of $\varphi=\pi / 2$ (moving up, green symbols) and $\varphi=3 \pi / 2$ (moving down, orange) along the spacecraft path across the second magnetic flux tube (see Figure 4). Vertical dashed lines are the magnetic boundaries of the flux tube. Note the spatial displacement between the two intensity profiles by $2 r_{g}(0.01 \mathrm{AU})$.

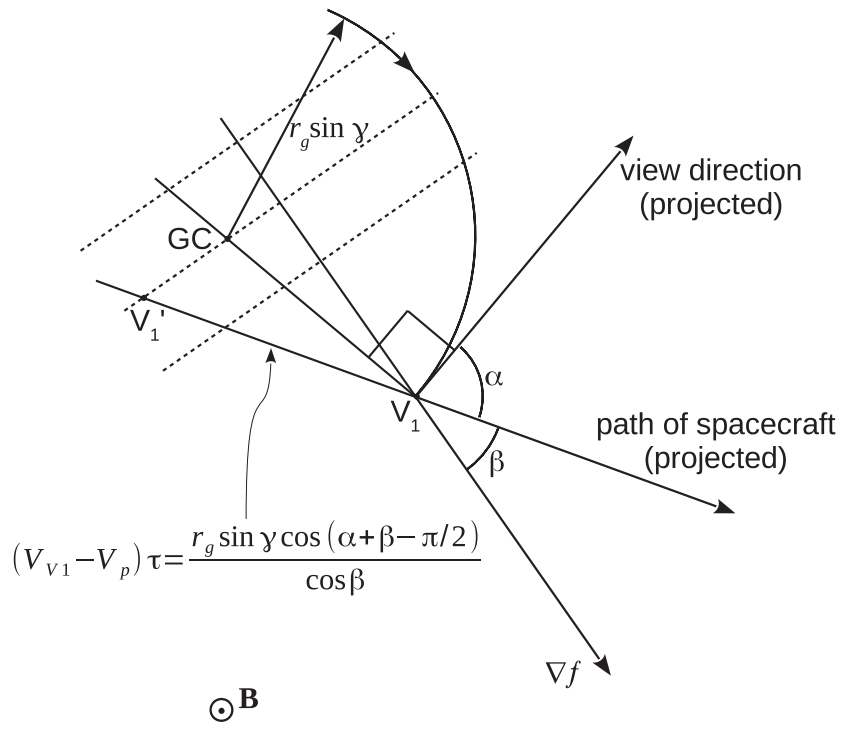

Figure 8. Geometry used to compute the time delay between LET telescopes. All vectors shown are projections onto a plane normal to the magnetic field. 
Table 2

LET Telescope Direction Vectors in the Spacecraft RTN Coordinate System, Angles Used in the Time Delay Calculation (see Figure 8), and Field of View Averaged Time Delays for Zero Plasma Radial Velocity

\begin{tabular}{lcccccccc}
\hline \hline & $R$ & $T$ & $N$ & $\gamma$ & $\alpha$ & $\beta$ & $\tau, \mathrm{hr}\left(T^{-3 / 2}\right)$ \\
\hline LET B & -0.596 & 0.686 & 0.417 & $131.8^{\circ}$ & $125.8^{\circ}$ & $0^{\circ}$ & 6.27 \\
LET D & -0.675 & -0.147 & -0.723 & $104.7^{\circ}$ & $229.3^{\circ}$ & $0^{\circ}$ & -7.61 & -8.79 \\
\hline
\end{tabular}

constant intensity plane passing through $\mathrm{GC}$ and $\left.\mathrm{V}_{1}^{\prime}\right)$ and the point where this change was actually measured $\left(\mathrm{V}_{1}\right)$ is

$$
\tau=\frac{r_{g} \sin \gamma \cos (\alpha+\beta-\pi / 2)}{\left(V_{V 1}-V_{p}\right) \cos \beta}
$$

where $V_{p}$ is the radial component of the plasma velocity.

For a typical heliosheath spectrum $J \sim T^{-3 / 2}$ the mean Larmor radius of protons between 2 and $8 \mathrm{MeV}$ corresponds to an energy of $3.84 \mathrm{MeV}$. In the dropouts lower energy particles are depleted more than those with high energy, so we also consider a harder $T^{-1}$ spectrum, for which the energy of a proton with the mean Larmor radius is $4.5 \mathrm{MeV}$. We also need to average (6) over the view angle of the instrument, to obtain

$$
\begin{aligned}
\tau= & \frac{\bar{r}_{g}}{\pi \sin ^{2} \theta_{\max }\left(V_{V 1}-V_{p}\right) \cos \beta} \\
& \times \int_{0}^{\theta_{\max }} \int_{0}^{2 \pi} \sin \gamma(\theta, \varphi) \cos [\alpha(\theta, \varphi) \\
& +\beta-\pi / 2] \cos \theta \sin \theta d \theta d \varphi,
\end{aligned}
$$

where $\bar{r}_{g}$ is the distribution-averaged Larmor radius and $\theta_{\max }=25^{\circ}$ is the LET aperture. The LET B and D telescopes are oriented in such a way that increasing $\beta$ leads to a reduction in $\tau$ for both (and vice versa); as a result, their difference is only weakly dependent on the angle $\beta$. The center-of-view directions of LET B and D, angles $\alpha, \beta$, and $\gamma$, and time delays computed using Equation (7) with $\beta=0$, zero plasma velocity, and two power-law spectra are given in Table 2. LET D is looking ahead along the path, so its time delay has a negative sign. The measured difference between the two sensors is $\tau_{B}-\tau_{D}$. Using the parameters in Table 2, this difference is $\sim 14-15 \mathrm{hr}$, which is quite close to that measured for the second flux tube encounter event (12-14 hours). Our result, therefore, is consistent with zero plasma radial velocity inside the flux tube.

We can also estimate the range of possible radial plasma speeds that would produce the observed time difference (13 hours on average) by varying the angle $\beta$ within certain limits. Figure 9 shows the dependence of $V_{p}$ on $\beta$ for two values of the spectral power law. We can see that even for a relatively wide range of directions of $\nabla f$, the deduced plasma speed is within a few kilometers per second from zero, with a higher chance of negative values. The delay between LET B and $\mathrm{D}$ inside the first flux tube (not shown) was similar in magnitude, which means that the radial plasma speed was also small 10-15 days earlier. This does not rule out more rapid plasma motion during times when the ion intensities changed only gradually. However, as discussed in the next section, these anisotropy measurements favor the flux tube crossing over the moving boundary hypothesis as the explanation for the particle dropout events.

\section{DISCUSSION}

As shown in this paper, a $10 \%$ per AU gradient in magnetic field strength in the latitudinal direction is sufficient to explain every major observed feature of heliospheric energetic ion populations at the heliopause crossed by Voyager 1 in 2012 August. The large second-order anisotropies and the dependence of the width of the intensity depletion past the $\mathrm{HC}$ on particle rigidity are all reproduced in our model. To simulate the dropouts, we included two cylindrical magnetic flux tubes that were originally part of the OHS, but became embedded in the IHS region as a result of the ballooning instability driven by a kinetic pressure gradient across the heliopause. If the conditions for the instability development are favorable, its growth rate would be similar to the duration of the boundary crossing events.

Our new results do not contradict or invalidate the model presented earlier (Florinski et al. 2013). For the sake of simplicity, the suppression of magnetic field line meandering at the boundaries of flux tubes and the $\mathrm{HC}$ as a result of shear in the flow, which was an important component of the previous paper, were modeled by decreasing the rate of field meandering in the entire OHS. As a result, the transient features in lower rigidity ions are more spread out, and the depths of the intensity dropouts in the model are somewhat less than observed. FLRW suppression would make these features sharper for streaming particles, but are not expected to affect higher rigidity particles, such as a few $\mathrm{MeV} / \mathrm{n}$ helium and oxygen singly charged ions, very much, because their cross-field transport is dominated by drift.

The heliosheath-invading flux tube hypothesis, originally proposed by Krimigis et al. (2013), appears to work well with our model, explaining the dropouts and subsequent particle recoveries. The flux tubes in our model are thin filaments with length-to-diameter ratios of several hundred. It is conceivable that the magnetic forces acting on the heliosheath plasma in the direction normal to the axes of the flux tubes are responsible for aligning the magnetic fields inside and outside the flux tubes. Our attempts to use alternative transport geometries, such as the one with alternating "slabs" of IHS and OHS plasma, were not successful, because they could not reproduce the nearly complete recoveries after each dropout. The flux tubes have a finite cross-section; thus, such a recovery is possible. There is still a small decrease from $0.4 \mathrm{AU}$ to the $\mathrm{HC}$, but this is also seen in observations.

The flux tube encounter and the associated sharp drops and subsequent recoveries of heliospheric energetic particles afforded a unique opportunity to infer the plasma speed from the Larmor radius crossing time (with the geometry of the LET telescopes taken into account). We found that the observed difference in the telescopes looking backward and forward along the spacecraft's trajectory are consistent with zero, or small (under $5 \mathrm{~km} \mathrm{~s}^{-1}$ ) negative background plasma velocity in the radial direction inside the flux tube(s). This is an independent indirect measurement of the plasma velocity near 


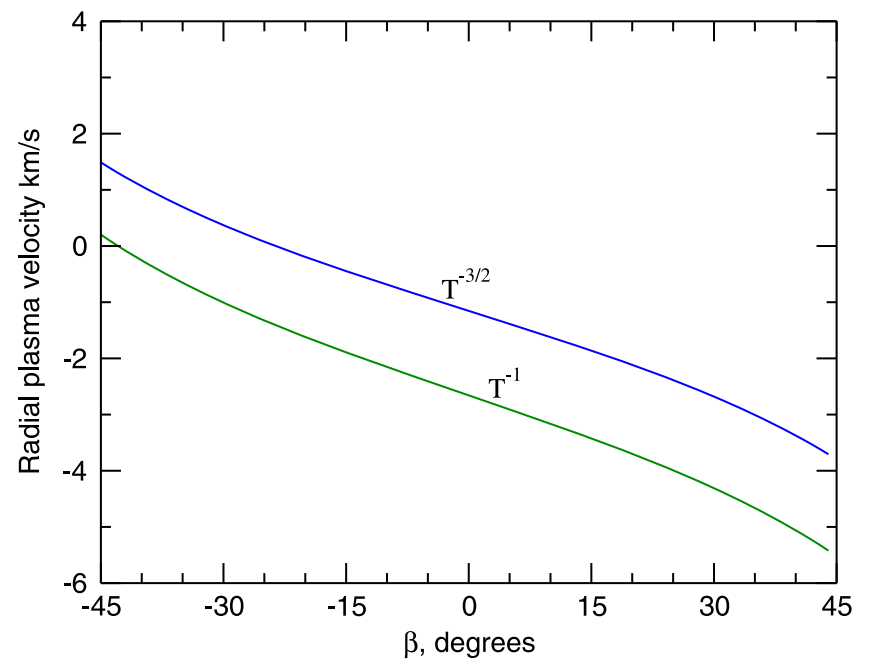

Figure 9. Radial plasma speed inside flux tube 2 calculated from the Larmor radius spacecraft crossing time as a function of the angle between the radial direction and the energetic particle intensity gradient for $J \sim T^{-3 / 2}$ (blue line) and $J \sim T^{-1}$ (green).

the heliopause, complementing the indirect measurements based on LECP ion anisotropy that yielded near zero radial velocity since the middle of 2010 (Krimigis et al. 2011).

If our theory is correct, Voyager 2 might see a very different heliospheric energetic particle behavior after it passes the heliopause. A northern gradient of magnetic field strength would prevent positive ions from drifting into interstellar space, in which case the ions would disappear immediately past the HC. However, a southern gradient is still possible if the gradient is of a local origin, associated with a disturbance on the surface of the heliopause, so that the higher rigidity gyrating particles would be observed for a period of time after the HC.

In a recent paper, Quenby \& Webber (2013) discussed several possible explanations for the particle intensity variations near the heliopause. In one of their models, the boundary was moving rapidly in and out past Voyager, producing dropouts in heliospheric ion intensities (see also Webber \& McDonald 2013). While we cannot exclude the possibility of rapid motion of the plasma during time intervals devoid of large particle intensity gradients, the anizotropy measurements reported here contradict the moving boundary interpretation of the dropout events. To see this, suppose that the heliopause moved sunward past Voyager, producing the intensity depletion, then reversed its direction and moved back beyond the spacecraft. In that case, the recovery would have occurred in reverse order (first in LET B, followed by LET D), contrary to what was observed. Furthermore, intensity at the bottom of the dropout in the LET D detector would have reached lower values than in LET B, because the former would be sampling farther into the depleted region. Instead, Figure 2 shows that the two intensities decreased by the same amount, consistent with the flux tube crossing scenario (see Figure 7).

Strauss et al. (2014) recently suggested that cross-field diffusion was the cause of large anisotropies of both ACRs and GCRs. Quenby \& Webber (2013) have also argued for a larger value of the perpendicular diffusion coefficient in the OHS. While the drift works remarkably well, cross field diffusion that depends on particle's charge per nucleon could potentially help differentiate between ACRs of different species. It is presently unknown whether there is enough field line meandering in the OHS on the scale of tens of AU (along the field) to produce a measurable effect. Regardless, we maintain that the Voyager 1 observation of heliospheric ions during the 2012 boundary crossing events are now mostly understood within the common transport framework. It remains to be seen whether GCRs are also amenable to this treatment or require an altogether different approach.

This work was supported, in part, by NASA grants NNX10AE46G, NNX11A064G, NNX12AH44G, NNX13AF99G, and NNX14AF43G, by NSF grant AGS0955700, and a cooperative agreement with NASA Marshall Space Flight Center NNM11AA01A. V.F. thanks Ed Roelof for a helpful discussion.

\section{REFERENCES}

Axford, W. I. 1965, P\&SS, 13, 115

Borovikov, S. N., \& Pogorelov, N. V. 2014, ApJL, 783, L16

Borovikov, S. N., Pogorelov, N. V., Zank, G. P., \& Kryukov, I. A. 2008, ApJ, 682, 1404

Burlaga, L. F., \& Ness, N. F. 2014, ApJ, 784, 146

Burlaga, L. F., Ness, N. F., Florinski, V., \& Heerikhuisen, J. 2014, ApJ, 792, 134

Burlaga, L. F., Ness, N. F., \& Stone, E. C. 2013, Sci, 341, 147

Burlaga, L. F., Ness, N. F., Gurnett, D. A., \& Kurth, W. S. 2013, ApJL, 778, L3

Florinski, V., Jokipii, J. R., Alouani-Bibi, F., \& le Roux, J. A. 2013, ApJL, 776, L37

Florinski, V., Zank, G. P., \& Pogorelov, N. V. 2005, JGR, 110, A07104

Gurnett, D. A., Kurth, W. S., Burlaga, L. F., \& Ness, N. F. 2013, Sci, 341,1489

Jokipii, J. R., \& Parker, E. N. 1969, ApJ, 155, 777

Krimigis, S. M., Decker, R. B., Roelof, E. C., et al. 2013, Sci, 341, 144

Krimigis, S. M., Roelof, E. C., Decker, R. B., \& Hill, M. E. 2011, Natur, 474,359

Parker, E. N. 1957, PhRv, 107, 924

Quenby, J. J., \& Webber, W. R. 2013, MNRAS, 436, 3306

Sakai, J.-I. 1982, ApJ, 263, 970

Shalchi, A. 2006, A\&A, 453, L43

Stone, E. C., Cummings, A. C., McDonald, F. B., et al. 2013, Sci, 341, 150

Stone, E. C., Vogt, R. E., McDonald, F. B., et al. 1977, SSRv, 21, 355

Strauss, R. D., \& Fichtner, H. 2014, A\&A, 572, L3

Strumik, M., Grzedzielski, S., Czechowski, A., Macek, W. M., \& Ratkiewicz, R. 2014, ApJL, 782, L7

Swisdak, M., Drake, J. F., \& Opher, M. 2013, ApJL, 774, L8

Viñas, A. F., \& Madden, T. R. 1986, JGR, 91, 1519

Webber, W. R., \& McDonald, F. B. 2013, GeoRL, 40, 1665 